The Be Phenomenon in Early-Type Stars, IAU Colloquium 175

ASP Conference Series, Vol. 214, 2000

M. A. Smith, H. F. Henrichs, and J. Fabregat, eds.

\title{
Paschen and Brackett Lines in Be stars
}

\section{Cidale}

Observatorio de La Plata, Paseo del Bosque S/N, 1900 La Plata, Argentina

J. Zorec, J.P. Maillard

Institut d'Astrophysique de Paris, CNRS, 98 bis bd. Arago, F-75014

Paris, France

N. Morrell

Observatorio de La Plata, Paseo del Bosque S/N, 1900 La Plata, Argentina

\begin{abstract}
The activities detected in Be stars indicate that the formation of the circumstellar envelope and its structure cannot be studied independently of the phenomena taking place in the outermost layers of the central stars. Assuming that related to the stellar activity there is an expanding atmospheric region with temperatures $T_{\mathrm{e}}>T_{\text {eff }}$ followed by an envelope with a decreasing temperature, we calculated hydrogen line profiles for different velocity fields and different positions of temperature maxima relative to the underlying photosphere. Results show that the $\mathrm{H} \alpha$ line is not very sensitive to changes introduced to the stellar atmosphere and to the nearby circumstellar layers. Moreover, the $\mathrm{H} \alpha$ emission line profiles look like those produced by disc-like circumstellar envelopes seen pole-on, although the model for the circumstellar envelope is spherical. However, the first members of the Paschen and Brackett series are strongly sensitive to any changes introduced in the photospheric and exophotospheric layers. We conclude that the study of these lines may then be valuable to obtain new insight on the activity of central stars and on the phenomena involved in circumstellar envelope formation in Be stars.
\end{abstract}

\section{Introduction}

All types of mass ejection discovered in Be stars, both continuous and discrete ones, which probably supply the mass necessary to build up their circumstellar envelopes (CE) are probably the outcome of strong activities in the outermost stellar layers. It is therefore possible that new information can be obtained on the active aspect of $\mathrm{CE}$ formation in $\mathrm{Be}$ stars if models of spectral lines related to them can be calculated. Apart from those lines of high ionized species and energy distribution in the far UV which provide information on layers with the lowest densities, it is worth studying transitions of abundant elements, which 

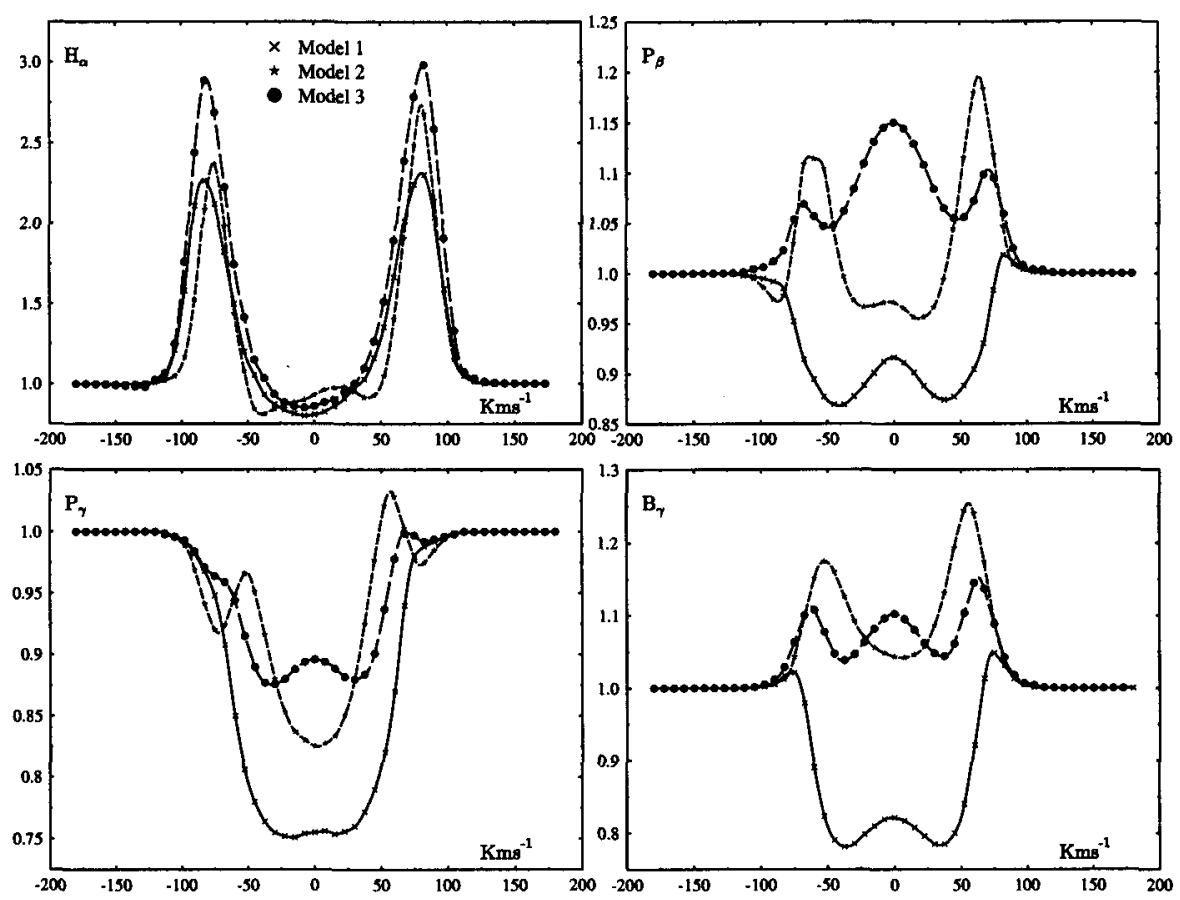

Figure 1. Model hydrogen $\mathrm{H} \alpha(6562 \AA), \mathrm{Pa} \beta(12818 \AA), \mathrm{Pa} \gamma(10938 \AA)$ and $\operatorname{Br} \gamma(21656 \AA)$ line profiles.

are also sensitive to temperature and density variations. Their formation region may concern not only the transition region between the star and the $\mathrm{CE}$, but probably also the stellar atmosphere itself.

\section{Hydrogen Paschen and Brackett lines}

Hydrogen is the most abundant element in stars. However, since the first studies of solar activity, it was realized that the $\mathrm{H} \alpha$ line, and in general most lines of the Balmer series, are "activity-blind" transitions (Thomas \& Athay 1961). This is due to the strongly radiation dominated source function of these transitions. Balmer lines may then be sensitive to conditions in layers where the radiative recombination rates are higher, ie. regions where the temperature is lower and/or the densities are higher $\left(R_{\mathrm{ki}} \propto N_{\mathrm{e}} T_{\mathrm{e}}^{-1 / 2}\right)$. In the same regions the intensity of Balmer lines depends on the extent of the region. Instead, the shape of the line profile depends on the velocity field in the transition region between the star and the CE, and thus on the mass-loss rate (Cidale \& Ringuelet 1993). Higher series in the hydrogen atom, like Paschen and Brackett series, have source functions with much stronger collisional domination than the Balmer series. It is expected then, that these lines may in some way be better indicators of activities taking place in layers near the stellar surface. Emission in Paschen lines of Be stars 
was observed up to very high members (Briot 1981; Andrillat et al. 1988). On the other hand, as this emission should respond to a rather strong population in levels higher than Balmer $(n=3,4)$ the emission is hence probably related to rather hot layers of the $\mathrm{CE}$. They may concern the star-CE transition region. In a preceding work (Chalabaev \& Maillard 1985) it was observed that the lower members of hydrogen Paschen and Brackett series are sensititve to velocity fields in the deep layers of the $\mathrm{CE}$ in $\gamma$ Cas. In the present contribution we wish to test the sensitivity of these same lines to the temperature structure in the exophotospheric layers and to compare it with that of the $\mathrm{H} \alpha$ line.

\section{The model of the CE}

To take into account the presence of a possible active region in the outermost stellar layers, as well as in the transition region star-CE, we used the following physical configuration. We assume the existence of an extended atmosphere which keeps unchanged the deepest layers of a classic stellar photosphere. Beyond the photosphere there is a region in radiative equilibrium at low expansion, followed by a hot and outwardly accelerating expanding zone. At distances $R \sim 2-3 R_{*}$ there is a drop of the temperature to an asymptotic low value $T_{o}$. Details of this model were given in (Cidale \& Ringuelet 1993). We also assume that the expanding region has a spherical geometry, where the velocity field increases monotonically outward up to an asymptotic value $V_{\max }$.

Here we present three models for the calculation of hydrogen lines, which differ from each other in the position of the maximum of a chromospheric-like temperature distribution. In particular, we selected velocity fields that yield $\mathrm{H} \alpha$ line profiles resembling those currently obtained from discs seen equator-on. These fields have low velocity gradients in the star-CE transition region. Moreover, the velocity fields were quite similar, as were the density distributions. The highest expansion velocity attained is $V_{\max }=120 \mathrm{~km} \mathrm{~s}^{-1}$. The supersonic branch of this velocity field begins at $R \sim 5 R_{*}$. The density and velocity distribution imply a mean mass-loss rate $\dot{M}=(1.8 \pm 0.5) \times 10^{-8} \mathrm{M}_{\odot} \mathrm{yr}^{-1}$. The position of the maximum temperature in the $\mathrm{CE}$ and/or transition region is parametrized with the parameter $\Delta_{1}: \Delta_{1}($ Model 1$)=1.0, \Delta_{1}($ Model 2$)=2.0$ and $\Delta_{1}$ (Model 3$)=3.0$, which correspond to the following positions of maxima respectively: $1.32 R_{*}, 1.64 R_{*}$ and $1.97 R_{*}$. The remaining common parameters in these models are $T_{\max }=25000 \mathrm{~K}, T_{\min }=12500 \mathrm{~K}$ and $T_{0}=5000 \mathrm{~K}$. The star underlying the CE was assumed to be characterized by $T_{\text {eff }}=20000 \mathrm{~K}$ and $\log g=3.0$.

The radiative transfer equation for accelerated expanding flows with spherical geometry is solved in the comoving frame, consistently with the statistical equilibrium equations for multilevel atoms (Mihalas \& Kunasz 1978). We considered the hydrogen atom with 10 levels, where the population was calculated in non-LTE. 


\section{Results}

Fig. 1 shows the results obtained for the hydrogen $\mathrm{H} \alpha(6562 \AA), \mathrm{Pa} \beta(12818 \AA)$, $\operatorname{Pa} \gamma(10938 \AA)$ and $\operatorname{Br} \gamma(21656 \AA)$ line profiles. The main conclusions we can draw are:

- The calculated $\mathrm{H} \alpha$ emission line profile is reminiscent of those due to disclike circumstellar envelopes seen equator-on. However, in our calculations we used spherical $\mathrm{CE}$ and there is a hot chromospheric-like region. The emission intensity increases slightly as the minimum of the chromospheric temperature distribution is more extended.

- Contrasting with the behaviour of the $\mathrm{H} \alpha$ line profile, which is almost insensitive to temperature changes, is the high variability detected in the first members of the Paschen and Brackett series as a function of the temperature maximum position. So, depending on the relative position of the temperature maximum to a given density distribution in the $\mathrm{CE}$, these lines can change from a profile dominated by absorptions to another of pure emission type.

- Comparing the intensities of calculated $\operatorname{Pa} \gamma$ and $\operatorname{Br} \gamma$ line profiles, we conclude that the Brackett series may possibly be more sensitive to temperature changes than the Paschen series.

- The emission in the Paschen and Brackett lines is higher as the maximum temperature is at larger distances from the central star. This reflects the fact that the region with high enough excitation to produce emission concerns a wider volume of matter. This also indicates the sensitivity of the studied lines to the high excitation of regions which are able to rise their emission.

\section{References}

Andrillat, Y., Jaschek, M., Jaschek, C. 1988, A\&AS 72, 129

Briot, D. 1981, A\&A 103, 5

Chalabaev, A.A., Maillard, J.P. 1985, ApJ 294, 640

Cidale, L., Ringuelet, A.E. 1993, ApJ 411, 874

Mihalas, D., Kunasz, P.B. 1978, ApJ 219, 635

Thomas, R.N., Athay, R. 1961, Physics of the Solar Chromosphere (New York: John Wiley Co.) 